\title{
Methamphetamine, 3,4-methylenedioxymethamphetamine (MDMA) and 3,4- methylenedioxypyrovalerone (MDPV) induce differential cytotoxic effects in bovine brain microvessel endothelial cells
}

\author{
Hector Rosas-Hernandez ${ }^{1}$, Elvis Cuevas ${ }^{1}$, Susan M. Lantz ${ }^{1}$, Kenner C. Rice ${ }^{2}$, Brenda M. \\ Gannon $^{3}$, William E. Fantegrossi ${ }^{3}$, Carmen Gonzalez ${ }^{4}$, Merle G. Paule ${ }^{1}$ and Syed F. Ali ${ }^{*}$. \\ ${ }^{1}$ Neurochemistry Laboratory, Division of Neurotoxicology, National Center for Toxicological \\ Research/FDA, Jefferson, AR, USA. ${ }^{2}$ Drug Design and Synthesis Section, Molecular Targets \\ and Medications Discovery Branch, NIDA/NIAAA, Bethesda, MD. ${ }^{3}$ Department of \\ Pharmacology \& Toxicology, UAMS, Little Rock, AR, USA. ${ }^{4}$ Facultad de Ciencias Quimicas, \\ UASLP, SLP, Mexico.
}

*Corresponding author:

Syed F. Ali, Ph.D.

Neurochemistry Laboratory,

Division of Neurotoxicology

National Center for Toxicological Research/FDA,

Jefferson, AR 72079, USA

Email: Syed.Ali@,fda.hhs.gov 


\section{Abstract}

Designer drugs such as synthetic psychostimulants are indicative of a worldwide problem. In additional to methamphetamine (METH), these drugs include 3,4- methylenedioxy-methamphetamine (MDMA) and commercial preparations of synthetic cathinones including 3,4-methylenedioxypyrovalerone (MDPV), typically referred to as "bath salts." These psychostimulants exert neurotoxic effects by altering monoamine systems in the brain. Additionally, METH and MDMA adversely affect the integrity of the blood-brain barrier (BBB): there are no current reports on the effects of MDPV on the BBB. The aim of this study was to compare the effects of METH, MDMA and MDPV on bovine brain microvessel endothelial cells (bBMVECs), an accepted in vitro model of the BBB. Confluent bBMVEC monolayers were treated with METH, MDMA and MDPV $(0.5 \mathrm{mM}-2.5 \mathrm{mM})$ for 24 hours. METH and MDMA increased LDH release only at the highest concentration $(2.5 \mathrm{mM})$, whereas MDPV induced cytotoxicity at all concentrations. MDMA and METH decreased cellular proliferation only at $2.5 \mathrm{mM}$, with similar effects observed after MDPV exposures starting at $1 \mathrm{mM}$. Only MDPV increased reactive oxygen species production at all concentrations tested whereas all 3 drugs increased nitric oxide production. Morphological analysis revealed different patterns of compound-induced cell damage. METH induced vacuole formation at $1 \mathrm{mM}$ and disruption of the monolayer at $2.5 \mathrm{mM}$. MDMA induced disruption of the endothelial monolayer from $1 \mathrm{mM}$ without vacuolization. On the other hand, MDPV induced monolayer disruption at doses $\geq 0.5 \mathrm{mM}$ without vacuole formation; at $2.5 \mathrm{mM}$, the few remaining cells lacked endothelial morphology. These data suggest that even though these synthetic psychostimulants alter monoaminergic systems, they each induce BBB toxicity by different mechanisms with MDPV being the most toxic.

Keywords: Methamphetamine, MDMA, MDPV, blood-brain barrier, cytotoxicity. 


\section{Introduction}

Drug abuse continues to be a worldwide problem. According to the United Nations Office on Drugs and Crime, as many as 315 million people have used illicit drugs [1]. Among these, the use of synthetic psychostimulants including methamphetamine (METH) and 3,4- methylenedioxy-methamphetamine (MDMA) account for more than 50 million people [1]. Both METH and MDMA induce neuropsychological effects via alteration of the brain dopamine (DA) and serotonin (5-HT) systems, promoting the release of both neurotransmitters from presynaptic neurons and prolonging the activation of both systems [2]. As a consequence, users experience a sense of euphoria and pleasure [3]. Unfortunately, both METH and MDMA induce DA and 5-HT terminal damage due to excitotoxicity, oxidative stress, and mitochondrial damage, resulting in depression, anxiety, memory impairment, psychosis and a higher risk of developing Parkinson's disease, among many other symptoms [2]. In the search for legal alternatives to the use of METH and MDMA new drugs are constantly being developed, including synthetic cathinones, also known as "bath salts" [4]. Among these substances, 3,4methylenedioxy-pyrovalerone (MDPV) is one one of the most prevalent [5]. MDPV has structural similarities with MDMA and also alters brain monoaminergic systems [4]. MDPV blocks the reuptake of DA [6] in a fashion similar to that of cocaine [7] but with greater potency [6], an effect also shared with METH and MDMA [2, 8, 9]. The collateral physiological effects of MDPV vary from agitation to seizures and cardiac arrest [10].

In addition to affecting monoaminergic systems, METH and MDMA induce neurotoxicity at least in part by increasing the permeability of the blood-brain barrier (BBB) [11]. The BBB is formed by continuous, non-fenestrated endothelial cells, surrounded by pericytes and astrocytes. The function of the BBB is to regulate the transit of substances into and out of the brain by maintaining low permeability and providing the proper chemical environment for adequate brain function [12]. The low permeability of the BBB is effected by tight junctions (TJ) between brain endothelial cells [12], but these TJs can be affected by a 
variety of molecules including reactive oxygen species (ROS) and nitric oxide (NO) [13]. In this regard, the toxic effects of METH on the BBB have been studied both in vitro [14, 15] and in vivo [16-19]. METH induces an increase in BBB permeability as evidenced using an in vitro model, bovine brain microvessel endothelial cells (bBMVECs). This effect is related to an increase in apoptosis and decrease in the expression of TJ proteins $[14,15]$. In vivo, METH also induces an increase in BBB permeability, also associated with a decrease in $\mathrm{TJ}$ proteins and increases in neuronal degeneration in the regions of BBB compromise [16, 17, 19]. Similar effects have been reported for MDMA, indicating that this drug also induces BBB toxicity that is accompanied by an increase in permeability $[20,21]$.

Despite the similarities in structure and biological activity of these three drugs, there are no published reports on the effect of MDPV on the BBB. The aim of this study was, thus, to evaluate the effects of MDPV on the BBB and compare them with the effects induced by METH and MDMA using bBMVECs as an in vitro model of the BBB.

\section{Materials and Methods}

\section{Chemicals}

S(+)-METH hydrochloride and S(+)-MDMA hydrochloride were purchased from Sigma Chemical Company (St. Louis, MO). Racemic MDPV was synthesized in the Drug Design and Synthesis Section at NIDA by Kenner C. Rice. The 5-Bromo-2'deoxy-uridine (BrdU) labeling and detection kit III and the cytotoxicity detection kit (LDH) were purchased from Roche Applied Sciences (Mannheim, Germany). Minimum Essential Medium Eagle (MEM) and Ham's F-12 Medium were purchased from Cellgro (Manassas, VA). All remaining media supplements and reagents were purchased from Sigma Chemical Company (St. Louis, MO).

\section{bBMVEC isolation}


bBMVECs were isolated from bovine brains as previously described [22]. Brains were obtained from the Cabot Meat Market in Cabot, AR and transported to the laboratory on ice-cold MEM medium pH 7.4. For isolation of the endothelial cells, the meninges were mechanically removed and the grey matter was collected and minced through a $100 \mu \mathrm{M}$ mesh screen. Dispase II enzyme $(5 \mathrm{mg} / \mathrm{mL})$ was added to the brain tissue and incubated for 3 hours at $37^{\circ} \mathrm{C}$. The tissue was then centrifuged (10 minutes at 9,170 x g) in a solution of $13 \%$ dextran to separate the crude capillary vessels which were collected and resuspended in collagenase/dispase enzyme $(5 \mathrm{mg} / \mathrm{mL})$ and incubated for 4 hours at $37{ }^{\circ} \mathrm{C}$. During the collagenase/dispase digestion, a percoll gradient was set up (60 minutes at 39,200 x g). The digested capillaries were centrifuged to remove the enzyme (10 minutes at 1,700 x g) and then placed into percoll gradient tubes and centrifuged to separate the bBMVECs (10 minutes at 1,700 x g), which were extracted from the percoll gradient and seeded into collagen-coated, fibronectin-treated plates in MEM-F12 media $+20 \%$ FBS and then placed into a humidified incubator $\left(37^{\circ} \mathrm{C}, 5 \% \mathrm{CO} 2\right)$ until confluence was reached, typically 8 -14 days.

\section{Treatments:}

bBMVECs were treated with increasing concentrations $(0.5 \mathrm{mM}-2.5 \mathrm{mM})$ of METH, MDMA or MVDP for 24 hours. Thimerasol was used as a positive control for cell death and sodium nitroprusside as a positive control for NO and ROS production.

\section{Cytotoxicity assay:}

Measurement of lactate dehydrogenase (LDH) activity released from the cytosol of damaged cells was measured in the supernatant as an index of necrosis. Briefly, cells were seeded in 96 -well plates at $85 \%$ confluence and treated with the drugs. After 24 hours, $100 \mu \mathrm{L}$ of the supernatant was collected and transferred to 96 well plates and a reaction mixture was added and incubated in the dark for 30 min at room temperature. LDH activity was quantified by measuring the absorbance at $490 \mathrm{~nm}[23,24]$. 


\section{Cellular proliferation:}

Cell proliferation was determined using a commercial bromodeoxyuridine (BrdU) incorporation kit according to the manufacturer's instructions. Briefly, BrdU was added to the bBMVECs at $70 \%$ confluence with or without the drug treatments and incubated for 24 hours. The BrdU-containing media was then removed and the cells were fixed for 30 minutes (ethanol 70\%/ $\mathrm{HCl} 25 \%, \mathrm{H}_{2} 05 \%$ ). Nucleases were added for 30 minutes to digest the BrdU-labeled DNA and then the samples were incubated for 30minutes with anti-BrdU-Peroxidase antibodies. Peroxidase substrate was then added and optical density measured at $405 \mathrm{~nm}$ (490 $\mathrm{nm}$ reference) to determine DNA synthesis and therefore proliferation.

\section{Nitric oxide production:}

NO production was quantified indirectly by measuring nitrites $\left(\mathrm{NO}_{2}\right)$ and nitrates $\left(\mathrm{NO}_{3}\right)$ [25]. Briefly, 100 $\mu \mathrm{L}$ aliquots of culture medium were transferred to a 96 well plate and incubated for $30 \mathrm{~min}$ in presence of $10 \mu \mathrm{L}$ of $\mathrm{N}-1$-napthylethylenediamine dihydrochloride $(0.1 \%, \mathrm{w} / \mathrm{v}), 10 \mu \mathrm{L}$ of sulfanilamide $(2 \%, \mathrm{w} / \mathrm{v})$ and $80 \mu \mathrm{L}$ of vanadium (III) chloride $(50 \mathrm{mM})$ at $37{ }^{\circ} \mathrm{C}$. After incubation, the optical density was measured at $560 \mathrm{~nm}$ to determine NO levels.

\section{Reactive Oxygen Species production:}

ROS production was determined by the oxidation of dichloro-dihydro-fluorescein diacetate (DCFH-DA) [26]. Briefly DCFH-DA at a final concentration of $100 \mu \mathrm{M}$ was added to the cells along with the various drug treatments. After 24 hours, fluorescence intensity was measured at $485 \mathrm{~nm}$ with a reference wavelength of $530 \mathrm{~nm}$. This assay measured oxidized DCF, therefore ROS production.

\section{Morphological Assessment:}

After 24 hours of drug exposure, bright field micrographs were taken at $20 \mathrm{X}$ magnification using a Nikon ECLIPSE Ti-E microscope. 


\section{Statistical analysis:}

Data were collected in triplicate from 3 independent experiments using cells obtained from 3 separate isolations and analyzed using one way ANOVA followed by post-hoc analysis. The data are presented as means \pm SEMs and the difference from control was considered significant if $p<0.05$.

\section{Results}

\section{METH, MDMA and MDPV induce necrosis}

METH and MDMA induced similar patterns of LDH release from bBMVECs, causing significant increases at only the $2.5 \mathrm{mM}$ concentration by 26.5 and $41.8 \%$, respectively. On the other hand, MDPV significantly increased LDH release at all concentrations: by $20.7 \%$ at $0.5 \mathrm{mM}, 37.0 \%$ at $1 \mathrm{mM}$ and $40.2 \%$ at $2.5 \mathrm{mM}$ (Fig. 1$)$.

\section{METH, MDMA and MDPV decrease cellular proliferation}

METH and MDMA also significantly decreased bBMVECs proliferation by $26.9 \%$ and $46.6 \%$, respectively, but only at $2.5 \mathrm{mM}$, whereas MDPV decreased cellular proliferation at 1 and $2.5 \mathrm{mM}$ by $47.1 \%$ and $74.0 \%$, respectively (Fig. 2).

\section{MDPV induces ROS production}

After 24 hours of exposure to METH, we did not observe any increase in ROS production and for MDMA, only the $0.5 \mathrm{mM}$ concentration was found to significantly increase ROS and only by $17.11 \%$. MDPV significantly increased ROS production by 30.6, 25.2 and $32.9 \%$ at $0.5 \mathrm{mM}, 1$ and $2.5 \mathrm{mM}$ respectively (Fig. 3).

\section{METH, MDMA and MDPV induce NO production}


METH significantly increased NO production by $73 \%$ but only at $2.5 \mathrm{mM}$. MDMA increased NO production by 57.2 and $61.7 \%$ at $0.5 \mathrm{mM}$ and $2.5 \mathrm{mM}$, respectively and MDPV increased NO levels by 65.4 and $86.7 \% \mu \mathrm{M}$ at 1 and $2.5 \mathrm{mM}$, respectively (Fig. 4).

\section{METH, MDMA and MDPV induce morphological changes}

Morphological analyses indicated that METH, MDMA, and MDPV all can induce morphological changes in the in vitro BBB model. The morphology of the control group was characterized by elongated cells forming a monolayer. $1 \mathrm{mM}$ METH induced slight vacuole formation (Fig. 5E), while $2.5 \mathrm{mM}$ METH induced disruption of the endothelial monolayer and increased vacuolization (Fig. 5H). MDMA slightly disrupted the monolayer at $1 \mathrm{mM}$ (Fig. 5F) and completely disrupted it at $2.5 \mathrm{mM}$ (Fig. 5G). MDPV also disrupted the endothelia cell monolayer at $0.5 \mathrm{mM}$ (Fig. 5D) and $1 \mathrm{mM}$ (Fig. 5G) and completely changed the endothelial cell morphology from elongated to round with only a few cells remaining attached at 2.5 $\mathrm{mM}$ (Fig. 5J).

\section{Discussion}

For the first time, the effects of MDPV on aspects of BBB integrity were investigated in this study. METH, MDMA and MDPV all induced cytotoxicity, but the toxicity induced by MDPV was observed at lower concentrations compared to those for METH and MDMA. METH-induced cytotoxicity and decreased cellular proliferation as previously reported by our group [15], as well as by others [14, 27, 28] was confirmed. Here only the $2.5 \mathrm{mM}$ METH concentration was shown to be cytotoxic for the brainderived endothelial cells; the same concentration of MDMA also decreased bBMVEC proliferation and promoted cytotoxicity, an effect that was not previously reported. One of the mechanisms reportedly associated with METH-induced BBB toxicity involves an increase in the production of ROS [27, 29]; however, we did not observe any increases in ROS production at any of the concentrations of METH used in the present study just the lowest concentration of MDMA. Despite the lack of ROS production after exposure to these drugs, an increase in NO production was observed 24 hours after treatment with 2.5 
mM of both METH and MDMA. Production of NO can also contribute to oxidative stress and lead to BBB impairment and toxicity [13]. METH induces an increase in BBB transcytosis and this is related to an increase in NO production in cultured brain endothelial cells [30]. Increases in NO levels induce changes in brain vascular tone and increase the permeability of the BBB [31,32]. Although not reported in the present study, previous reports have shown that both METH and MDMA can induce increases in BBB permeability $[15,17,20,21]$ : the effects reported here, including production of NO, cytotoxicity, decreased cellular proliferation, and disruption of the endothelial monolayer, may be involved in that effect.

In the present study, MDPV was shown to induce cytotoxicity, decrease cellular proliferation, and increase NO production at concentrations lower than those required for METH and MDMA, and to increase ROS production at all of the concentrations tested. Previous reports indicated that MDPV binds to the DA transporter with higher affinity than METH and is a more potent DA transporter inhibitor than METH [33]. Adverse effects of MDPV on aspects of BBB integrity have not been previously reported and we report here that it is more toxic than both METH and MDMA. One study did report on the transport of various drugs across an in vitro model of the BBB. It did indicate that MDPV crossed the BBB with a permeability ratio higher than that for many other synthetic cathinones and higher than that for METH [33]. Unlike METH and MDMA, there are no reports on the effects of MDPV on the permeability of the BBB. However, different studies have reported that METH, MDMA, and MDPV all induce similar effects on the vasculature [34-38]. For instance, in association with NO signaling [17] METH induces cerebral blood vessel vasoconstriction [35] and a reduction in cerebral blood flow [36] . MDMA induces coronary [38] and peripheral [39] vasoconstriction, whereas MDPV also induces peripheral [34] and integumentary vasoconstriction, as well as an increase in brain temperature, effects that suggest vasoconstriction of cerebral blood vessels [37]. These observations, along with data from the present study suggest that MDPV affects the brain vasculature and most likely increases the permeability of the BBB in a fashion similar to that for METH and MDMA. 


\section{Conclusion}

In summary, the findings from this study indicate that METH, MDMA and MDPV are all cytotoxic to bBMVECs and that the toxicity is dependent upon dose. This is the first study to report such effects for MDPV. The cytotoxic effects of MDPV occur at lower concentrations than for either METH or MDMA, demonstrating that it is more potent in causing toxicity in the in vitro BBB model used here. In addition, the mechanism(s) underlying the cause of damage may be different. Further investigations are required to confirm these observations and to elucidate the mechanisms involved.

\section{Acknowledgment}

This research was supported in part by an appointment (H. R-H.) to the Research Participation Program at the National Center for Toxicological Research administered by the Oak Ridge Institute for Science and Education through an interagency agreement between the U.S. Department of Energy and the U.S. Food and Drug Administration. NCTR Experiment E7584.01. A portion of this work was supported by the Intramural Research Programs of the National Institute on Drug Abuse and the National Institute on Alcoholism and Alcohol Abuse. The content is solely the responsibility of the authors and does not necessarily represent the official views of the US. Food and Drug Administration, the National Institute

on Drug Abuse, the National Institute of Alcohol Abuse and Alcoholism, or the National Institutes of Health. 


\section{Figure Captions}

Figure 1.- METH, MDMA and MDPV induce cytotoxicity. bBMVECs were treated with increasing concentrations of METH, MDMA or MDPV $(0.5 \mathrm{mM}-2.5 \mathrm{mM})$ for 24 hours. LDH release was taken as an index of cytotoxicity. Data represent the mean values \pm SEMs from three independent experiments conducted using triplicate samples. ${ }^{*} \mathrm{p}<0.05, * * * \mathrm{p}<0.001$ vs control.

Figure 2.- METH, MDMA and MDPV decrease cellular proliferation. bBMVECs were treated with increasing concentrations of METH, MDMA or MDPV $(0.5 \mathrm{mM}-2.5 \mathrm{mM})$ for 24 hours. BrdU incorporation was taken as an index of cellular proliferation. Data represent the mean values \pm SEMs from three independent experiments conducted using triplicate samples. ${ }^{*} \mathrm{p}<0.05, * * * \mathrm{p}<0.001$ vs control.

Figure 3.- MDMA and MDPV increase ROS production. bBMVECs were treated with increasing concentrations of METH, MDMA and MDPV (0.5 mM-2.5 mM) for 24 hours. DCFH-DA oxidation was taken as an index of ROS production. Data represent the mean values \pm SEMs from three independent experiments conducted using triplicate samples. ${ }^{*} \mathrm{p}<0.05, * * * \mathrm{p}<0.001$ vs control.

Figure 4.- METH, MDMA and MDPV increase NO production. bBMVECs were treated with increasing concentrations of METH, MDMA and MDPV (0.5 mM-2.5 mM) for 24 hours. The $\mathrm{NO}_{2} / \mathrm{NO}_{3}$ ration was taken as an index of NO production. Data represent the mean values \pm SEMs from three independent experiments conducted using triplicate samples. ${ }^{*} \mathrm{p}<0.05,{ }^{* *} \mathrm{P}<0.01, * * * \mathrm{p}<0.001$ vs control.

Figure 5.- METH, MDMA and MDPV induce morphological changes. bBMVECs were treated with increasing concentrations of METH, MDMA and MDPV (0.5 mM-2.5 mM) for 24 hours. Bright field images were taken at 20X magnifications. Panels show micrographs of control cells (A); METH-treated 
cells at $0.5 \mathrm{mM}(\mathrm{B}), 1 \mathrm{mM}(\mathrm{E})$ and $2.5 \mathrm{mM}(\mathrm{H})$; MDMA-treated cells at $0.5 \mathrm{mM}(\mathrm{C}), 1 \mathrm{mM}(\mathrm{F})$ and 2.5 $\mathrm{mM}(\mathrm{I})$ and MDPV-treated cells at $0.5 \mathrm{mM}(\mathrm{D}), 1 \mathrm{mM}(\mathrm{G})$ and $2.5 \mathrm{mM}(\mathrm{J})$.

\section{References}

[1] L. Burns, World Drug Report 2013 United Nations Office on Drugs and Crime, Drug Alcohol Rev, 33 (2014) 216-216.

[2] L.E. Halpin, S.A. Collins, B.K. Yamamoto, Neurotoxicity of methamphetamine and 3,4methylenedioxymethamphetamine, Life Sci, 97 (2014) 37-44.

[3] J.L. Cadet, V. Bisagno, C.M. Milroy, Neuropathology of substance use disorders, Acta Neuropathol, 127 (2014) 91-107.

[4] L. Karila, B. Megarbane, O. Cottencin, M. Lejoyeux, Synthetic cathinones: a new public health problem, Curr Neuropharmacol, 13 (2015) 12-20.

[5] A. Schneir, B.T. Ly, K. Casagrande, M. Darracq, S.R. Offerman, S. Thornton, C. Smollin, R. Vohra, C. Rangun, C. Tomaszewski, R.R. Gerona, Comprehensive analysis of "bath salts" purchased from California stores and the internet, Clin Toxicol (Phila), 52 (2014) 651-658.

[6] M.H. Baumann, J.S. Partilla, K.R. Lehner, E.B. Thorndike, A.F. Hoffman, M. Holy, R.B. Rothman, S.R. Goldberg, C.R. Lupica, H.H. Sitte, S.D. Brandt, S.R. Tella, N.V. Cozzi, C.W. Schindler, Powerful Cocaine-Like Actions of 3,4-Methylenedioxypyrovalerone (MDPV), a Principal Constituent of Psychoactive 'Bath Salts' Products, Neuropsychopharmacol, 38 (2013) 552-562.

[7] V. Verma, Classic Studies on the Interaction of Cocaine and the Dopamine Transporter, Clin Psychopharmacol Neurosci, 13 (2015) 227-238.

[8] A.E. Fleckenstein, R.R. Metzger, J.W. Gibb, G.R. Hanson, A rapid and reversible change in dopamine transporters induced by methamphetamine, Eur J Pharmacol, 323 (1997) R9-10.

[9] V. Sandoval, E.L. Riddle, Y.V. Ugarte, G.R. Hanson, A.E. Fleckenstein, Methamphetamineinduced rapid and reversible changes in dopamine transporter function: An in vitro model, $J$ Neurosci, 21 (2001) 1413-1419.

[10] O. Beck, L. Franzen, M. Backberg, P. Signell, A. Helander, Intoxications involving MDPV in Sweden during 2010-2014: Results from the STRIDA project, Clin Toxicol (Phila), 53 (2015) 865-873.

[11] E. O'Shea, A. Urrutia, A.R. Green, M.I. Colado, Current preclinical studies on neuroinflammation and changes in blood-brain barrier integrity by MDMA and methamphetamine, Neuropharmacology, 87 (2014) 125-134.

[12] B.W. Chow, C. Gu, The molecular constituents of the blood-brain barrier, Trends Neurosci, 38 (2015) 598-608.

[13] R. Moretti, J. Pansiot, D. Bettati, N. Strazielle, J.F. Ghersi-Egea, G. Damante, B. Fleiss, L. Titomanlio, P. Gressens, Blood-brain barrier dysfunction in disorders of the developing brain, Front Neurosci, 9 (2015) 40.

[14] N.U. Parikh, R. Aalinkeel, J.L. Reynolds, B.B. Nair, D.E. Sykes, M.J. Mammen, S.A. Schwartz, S.D. Mahajan, Galectin-1 suppresses methamphetamine induced neuroinflammation in human brain microvascular endothelial cells: Neuroprotective role in maintaining blood brain barrier integrity, Brain Res, 1624 (2015) 175-187. 
[15] H. Rosas-Hernandez, E. Cuevas, S.M. Lantz-McPeak, S.F. Ali, C. Gonzalez, Prolactin protects against the methamphetamine-induced cerebral vascular toxicity, Curr Neurovasc Res, 10 (2013) 346-355.

[16] J.F. Bowyer, S. Ali, High doses of methamphetamine that cause disruption of the bloodbrain barrier in limbic regions produce extensive neuronal degeneration in mouse hippocampus, Synapse, 60 (2006) 521-532.

[17] H.S. Sharma, S.F. Ali, Alterations in blood-brain barrier function by morphine and methamphetamine, Ann N Y Acad Sci, 1074 (2006) 198-224.

[18] J.F. Bowyer, B. Robinson, S. Ali, L.C. Schmued, Neurotoxic-related changes in tyrosine hydroxylase, microglia, myelin, and the blood-brain barrier in the caudate-putamen from acute methamphetamine exposure, Synapse, 62 (2008) 193-204.

[19] T. Martins, S. Baptista, J. Goncalves, E. Leal, N. Milhazes, F. Borges, C.F. Ribeiro, O. Quintela, E. Lendoiro, M. Lopez-Rivadulla, A.F. Ambrosio, A.P. Silva, Methamphetamine transiently increases the blood-brain barrier permeability in the hippocampus: role of tight junction proteins and matrix metalloproteinase-9, Brain Res, 1411 (2011) 28-40.

[20] A. Rubio-Araiz, M. Perez-Hernandez, A. Urrutia, F. Porcu, E. Borcel, M.D. Gutierrez-Lopez, E. O'Shea, M.I. Colado, 3,4-Methylenedioxymethamphetamine (MDMA, ecstasy) disrupts bloodbrain barrier integrity through a mechanism involving P2X(7) receptors, Int J Neuropsychoph, 17 (2014) 1243-1255.

[21] H.S. Sharma, S.E. Ali, Acute Administration of 3,4-Methylenedioxymethamphetamine Induces Profound Hyperthermia, Blood-Brain Barrier Disruption, Brain Edema Formation, and Cell Injury An Experimental Study in Rats and Mice Using Biochemical and Morphologic Approaches, Ann Ny Acad Sci, 1139 (2008) 242-258.

[22] K.L. Audus, R.T. Borchardt, Bovine brain microvessel endothelial cell monolayers as a model system for the blood-brain barrier, Ann N Y Acad Sci, 507 (1987) 9-18.

[23] T. Decker, M.L. Lohmann-Matthes, A quick and simple method for the quantitation of lactate dehydrogenase release in measurements of cellular cytotoxicity and tumor necrosis factor (TNF) activity, J Immunol Methods, 115 (1988) 61-69.

[24] M. Rayamajhi, Y. Zhang, E.A. Miao, Detection of pyroptosis by measuring released lactate dehydrogenase activity, Methods Mol Biol, 1040 (2013) 85-90.

[25] K.M. Miranda, M.G. Espey, D.A. Wink, A rapid, simple spectrophotometric method for simultaneous detection of nitrate and nitrite, Nitric Oxide, 5 (2001) 62-71.

[26] H. Wang, J.A. Joseph, Quantifying cellular oxidative stress by dichlorofluorescein assay using microplate reader, Free Radic Biol Med, 27 (1999) 612-616.

[27] X. Zhang, A. Banerjee, W.A. Banks, N. Ercal, N-Acetylcysteine amide protects against methamphetamine-induced oxidative stress and neurotoxicity in immortalized human brain endothelial cells, Brain Res, 1275 (2009) 87-95.

[28] J.W. Seo, S.M. Jones, T.A. Hostetter, J.J. Iliff, G.A. West, Methamphetamine induces the release of endothelin, J Neurosci Res, 94 (2016) 170-178.

[29] S.H. Ramirez, R. Potula, S. Fan, T. Eidem, A. Papugani, N. Reichenbach, H. Dykstra, B.B. Weksler, I.A. Romero, P.O. Couraud, Y. Persidsky, Methamphetamine disrupts blood-brain barrier function by induction of oxidative stress in brain endothelial cells, J Cereb Blood Flow Metab, 29 (2009) 1933-1945.

[30] T. Martins, T. Burgoyne, B.A. Kenny, N. Hudson, C.E. Futter, A.F. Ambrosio, A.P. Silva, J. Greenwood, P. Turowski, Methamphetamine-induced nitric oxide promotes vesicular transport in blood-brain barrier endothelial cells, Neuropharmacology, 65 (2013) 74-82.

[31] A.T. Argaw, L. Asp, J. Zhang, K. Navrazhina, T. Pham, J.N. Mariani, S. Mahase, D.J. Dutta, J. Seto, E.G. Kramer, N. Ferrara, M.V. Sofroniew, G.R. John, Astrocyte-derived VEGF-A drives blood-brain barrier disruption in CNS inflammatory disease, J Clin Invest, 122 (2012) 24542468. 
[32] O.Z. Chi, C. Hunter, X. Liu, H.R. Weiss, Effects of VEGF and nitric oxide synthase inhibition on blood-brain barrier disruption in the ischemic and non-ischemic cerebral cortex, Neurol Res, 27 (2005) 864-868.

[33] L.D. Simmler, T.A. Buser, M. Donzelli, Y. Schramm, L.H. Dieu, J. Huwyler, S. Chaboz, M.C. Hoener, M.E. Liechti, Pharmacological characterization of designer cathinones in vitro, $\mathrm{Br} \mathrm{J}$ Pharmacol, 168 (2013) 458-470.

[34] E.A. Kiyatkin, A.H. Kim, K.T. Wakabayashi, M.H. Baumann, Y. Shaham, Effects of social interaction and warm ambient temperature on brain hyperthermia induced by the designer drugs methylone and MDPV, Neuropsychopharmacol, 40 (2015) 436-445.

[35] S.M. Kousik, T.C. Napier, R.D. Ross, D.R. Sumner, P.M. Carvey, Dopamine receptors and the persistent neurovascular dysregulation induced by methamphetamine self-administration in rats, J Pharmacol Exp Ther, 351 (2014) 432-439.

[36] O. Polesskaya, J. Silva, C. Sanfilippo, T. Desrosiers, A. Sun, J. Shen, C. Feng, A. Polesskiy, R. Deane, B. Zlokovic, K. Kasischke, S. Dewhurst, Methamphetamine causes sustained depression in cerebral blood flow, Brain Res, 1373 (2011) 91-100.

[37] K.T. Wakabayashi, S.E. Ren, E.A. Kiyatkin, Methylenedioxypyrovalerone (MDPV) mimics cocaine in its physiological and behavioral effects but induces distinct changes in NAc glucose, Front Neurosci, 9 (2015) 324.

[38] K.E. Baker, A.A. Herbert, K.J. Broadley, Vasoconstriction of porcine left anterior descending coronary artery by ecstasy and cathinone is not an indirect sympathomimetic effect, Vasc Pharmacol, 47 (2007) 10-17.

[39] E.A. Kiyatkin, A.H. Kim, K.T. Wakabayashi, M.H. Baumann, Y. Shaham, Critical role of peripheral vasoconstriction in fatal brain hyperthermia induced by MDMA (Ecstasy) under conditions that mimic human drug use, J Neurosci, 34 (2014) 7754-7762. 
Figure 1

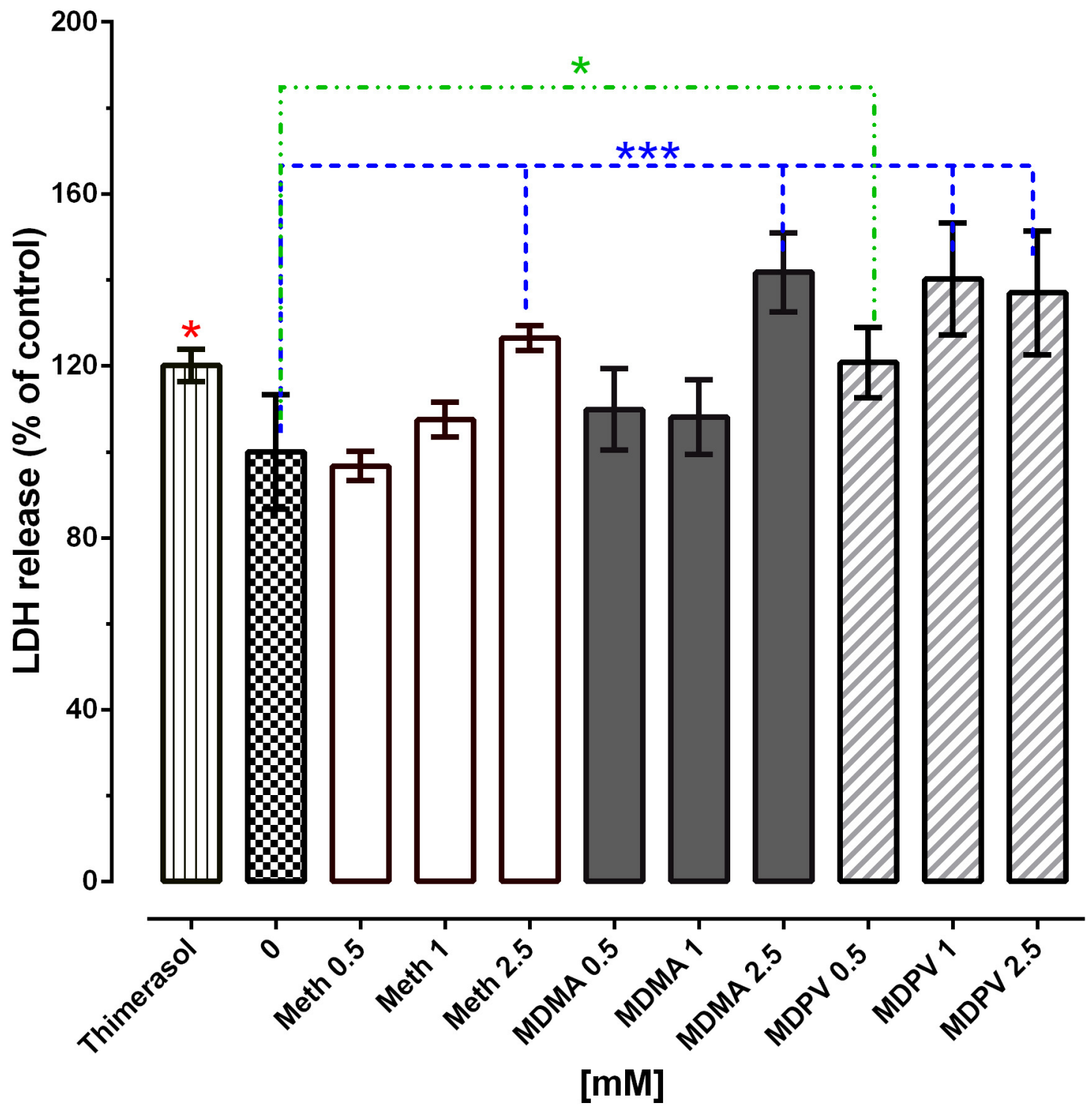


Figure 2

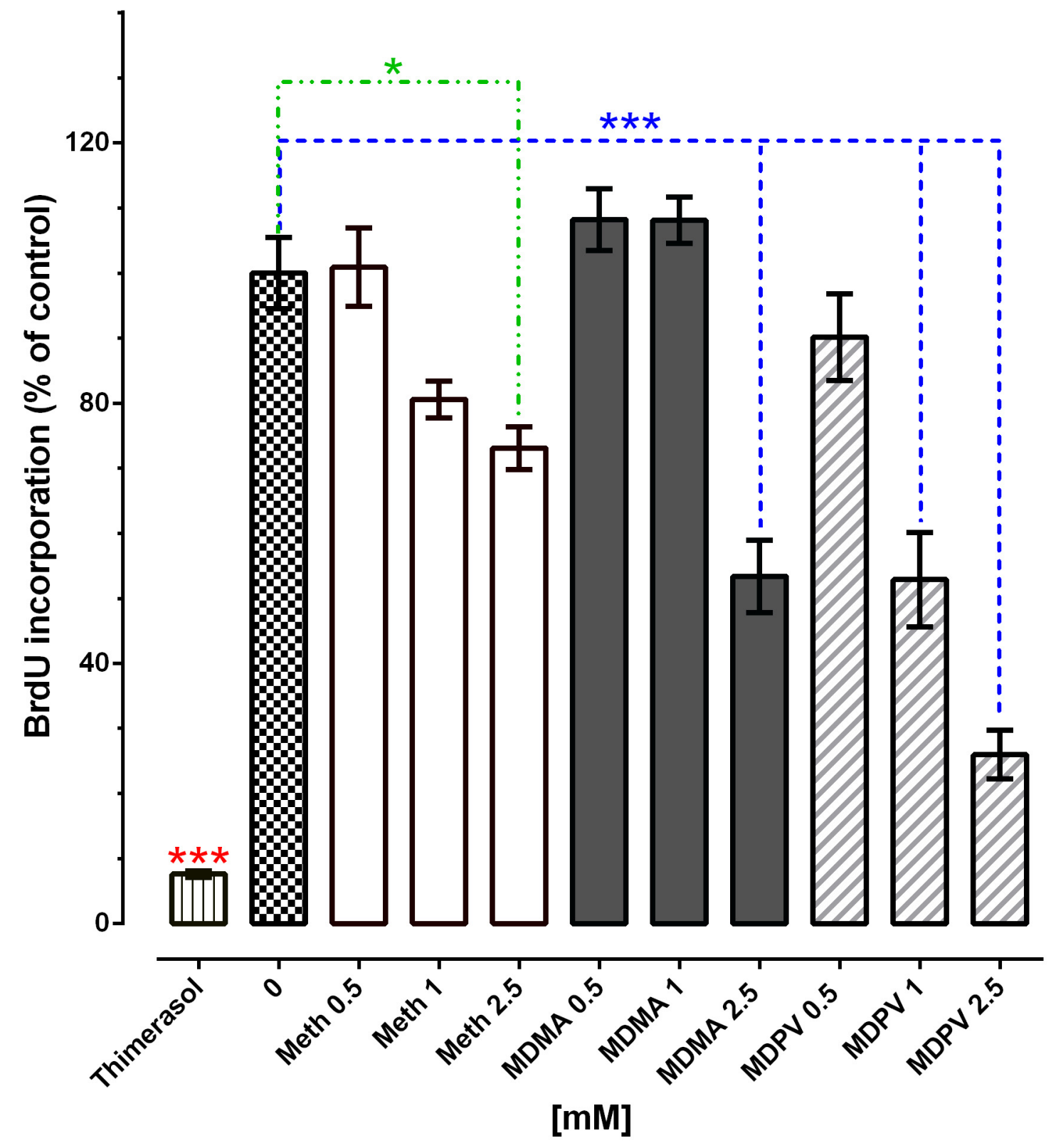


Figure 3

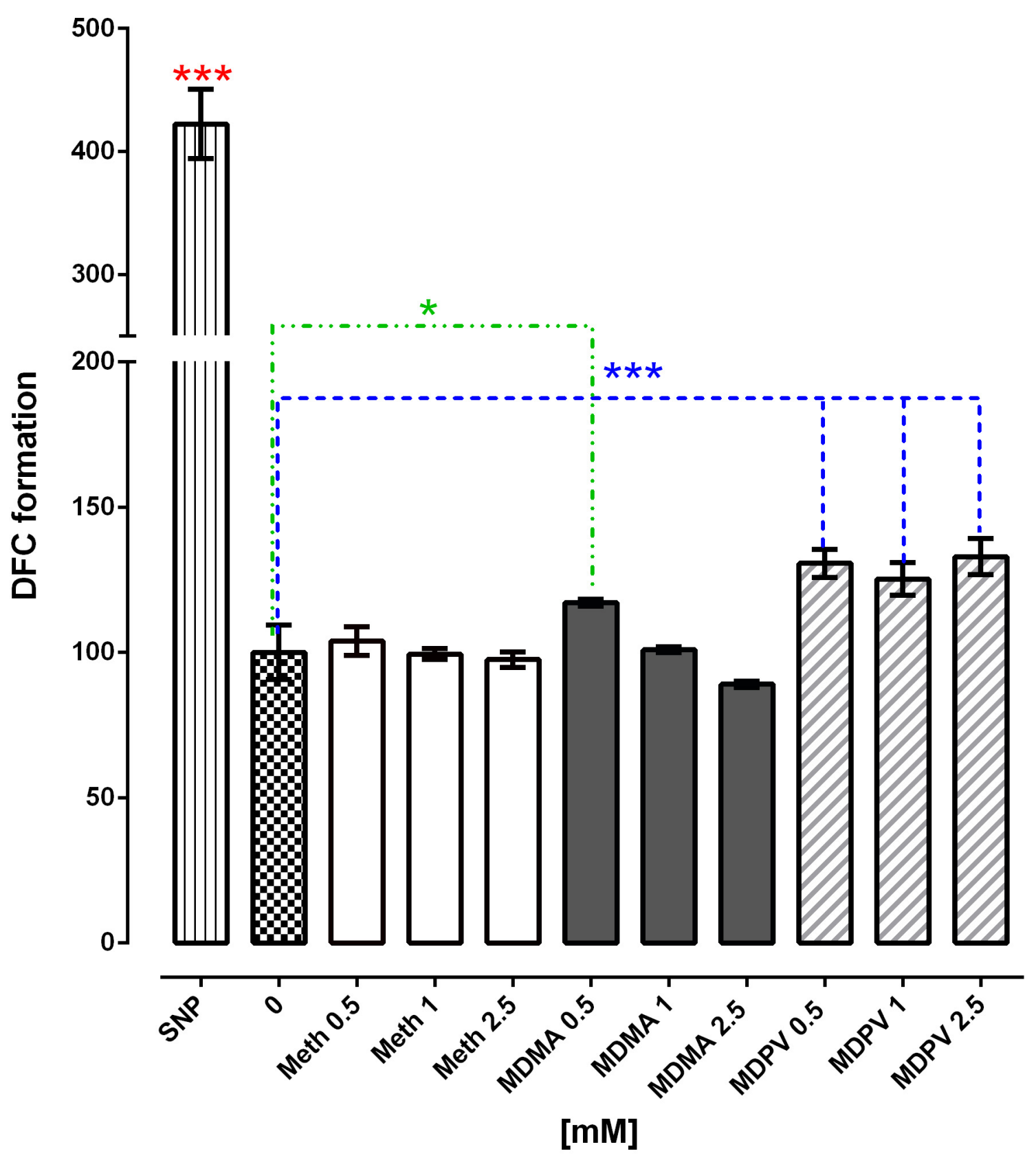


Figure 4

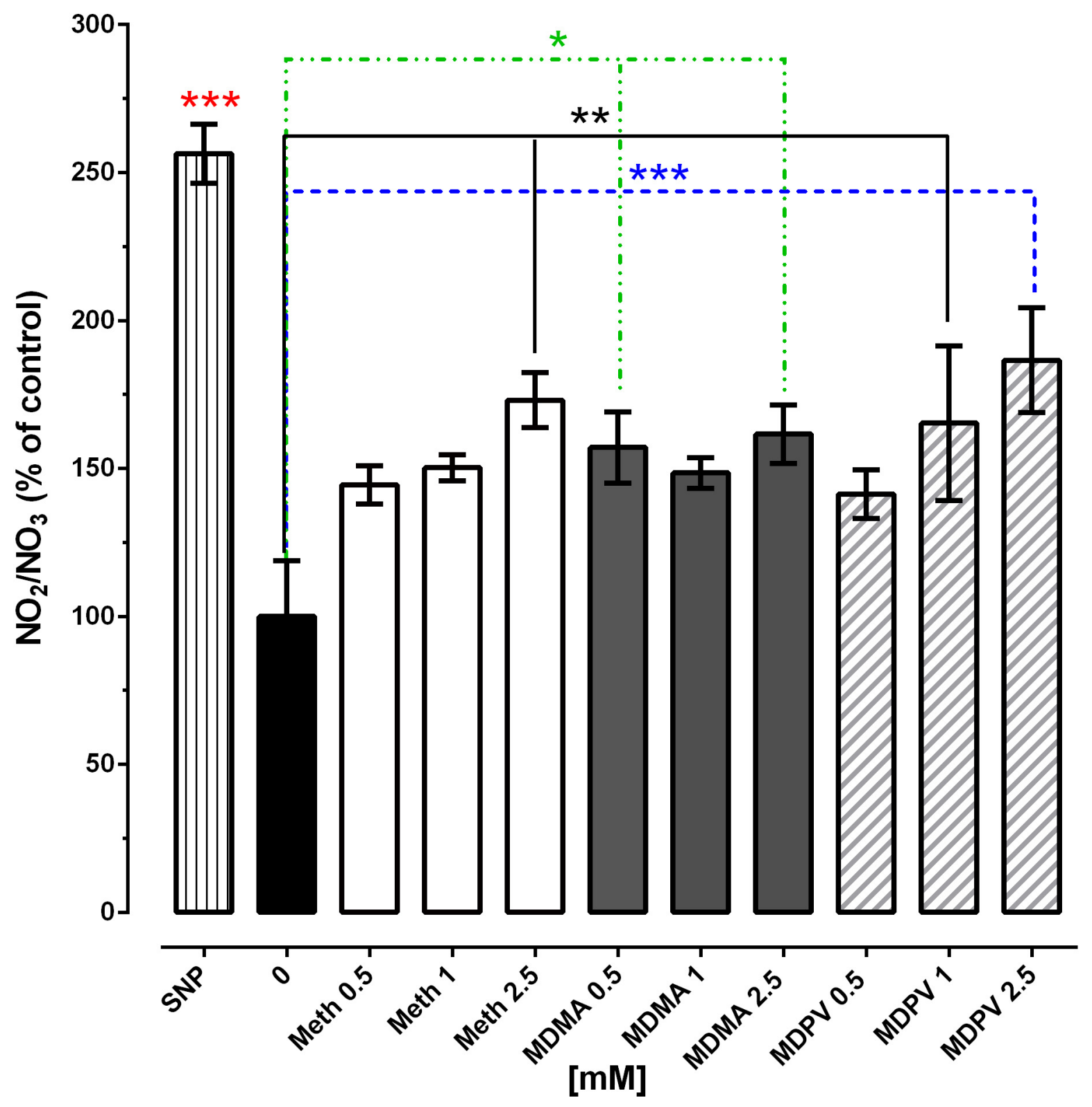


Figure 5

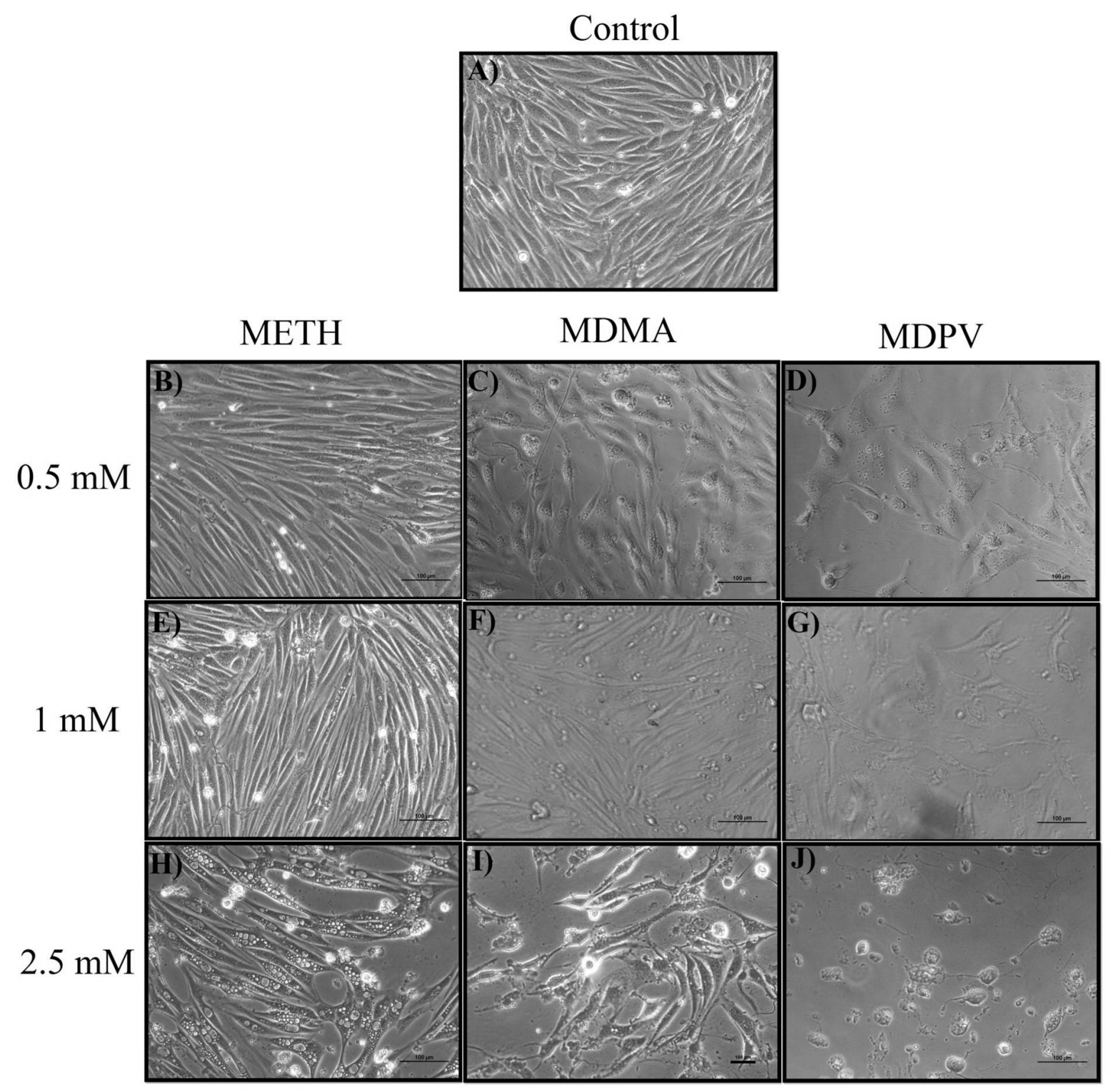

